\title{
Quantum Monte Carlo study of a nonmagnetic impurity in the two-dimensional Hubbard model
}

\author{
N. Bulut \\ Department of Physics, Koç University, Sariyer, 34450 Istanbul, Turkey
}

(Received 6 August 2003; published 9 December 2003)

\begin{abstract}
In order to investigate the effects of nonmagnetic impurities in strongly correlated systems, quantum Monte Carlo (QMC) simulations have been carried out for the doped two-dimensional Hubbard model with one nonmagnetic impurity. Using a bare impurity potential which is onsite and attractive, magnetic and singleparticle properties have been calculated. The QMC results show that giant oscillations develop in the Knight shift response around the impurity site due to the short-range antiferromagnetic correlations. These results are useful for interpreting the NMR data on Li- and Zn-substituted layered cuprates.
\end{abstract}

DOI: 10.1103/PhysRevB.68.235103 PACS number(s): 71.10.Fd, 02.70.Ss, 76.60.-k, 74.72.Bk

\section{INTRODUCTION}

The substitution of impurities into strongly correlated systems is a useful probe of the intrinsic electronic correlations. For instance, in the layered cuprates it has been found that the substitution of nonmagnetic impurities in place of $\mathrm{Cu}$ in the $\mathrm{CuO}_{2}$ planes strongly influences the superconducting ${ }^{1}$ and the magnetic correlations. ${ }^{2-7}$ These experiments have provided valuable insight into the interplay of the magnetic, superconducting, and density correlations in these materials. In particular, the NMR experiments find that when a uniform magnetic field is applied, the electronic spins in the $\mathrm{CuO}_{2}$ planes in $\mathrm{YBa}_{2} \mathrm{Cu}_{3} \mathrm{O}_{6+x}$ polarize to form an oscillatory pattern which decays away from the impurities. ${ }^{2-7}$ These are observed as giant oscillations in the Knight shift response of the system. Furthermore, the Knight shifts for nuclei close to the impurity have a perfect Curie-Weiss temperature dependence. The origin of these behaviors is an important question.

Theoretically, the problem of a nonmagnetic impurity in strongly correlated systems such as the two-dimensional (2D) $t-J$ and Hubbard models have been studied using various many-body techniques. The single-particle properties of an impurity in 2D $t-J$ clusters were studied with the exact diagonalization technique., ${ }^{8,9}$ The magnetic and singleparticle correlations around a nonmagnetic impurity in the doped 2D Hubbard model were studied with the slave boson and the quantum Monte Carlo (QMC) techniques. ${ }^{10}$ These calculations have found Friedel oscillations in the electron density. There are also RPA-type calculations of the Knight shifts for a nonmagnetic impurity in the 2D Hubbard model, ${ }^{11-13}$ which suggest that the locally enhanced antiferromagnetic $(\mathrm{AF})$ correlations around the impurity are responsible for the giant oscillations and the Curie-Weiss behavior observed in the Knight shift measurements.

In this paper, the magnetic and single-particle correlations around one nonmagnetic impurity in the Hubbard lattice will be studied with the QMC technique. For this purpose, the determinantal QMC algorithm introduced by Blankenbecler, Scalapino, and Sugar $^{14}$ and described by Ref. 15 will be used. The emphasis in this paper will be on the Knight shift response of the system in the metallic state near half filling. The QMC data show that as short-range AF fluctuations grow, giant oscillations develop in the Knight shift response.
Here, the real-space structure of these oscillations as well as the pattern in the electron density will be discussed. In addition, the effective impurity potential $V_{\text {eff }}$ will be extracted from the QMC data on the single-particle Green's function. It will be seen that $V_{\text {eff }}$ is weakly attractive at the sites neighboring the impurity. Because of the "fermion sign problem," the QMC calculations cannot be performed at sufficiently low temperatures where direct comparisons with the NMR data can be made. Instead, the QMC data will be compared with RPA-type calculations used for fitting the Knight shift data. ${ }^{12}$ It will be seen that the real-space patterns of the Knight shift, the electron density, and the effective impurity interaction obtained with the QMC technique are in good agreement with the results found in the RPA analysis of the Knight shift data. These emphasize the role of the AF fluctuations in producing the unusual Knight shift data on $\mathrm{Li}$ and $\mathrm{Zn}$ substituted $\mathrm{YBa}_{2} \mathrm{Cu}_{3} \mathrm{O}_{6+x}$. An important feature of these calculations is that here the system is always in the paramagnetic state, and no AF droplets have formed around the impurity.

In the following, the model used for a nonmagnetic impurity in the Hubbard model will be discussed. Then, the numerical data on the Knight shift and the local electron density will be presented. Next, these results will be compared with the previous RPA analysis of the Knight shift measurements. Finally, the QMC data on the effective impurity potential will be presented.

\section{MODEL}

The 2D Hubbard model is defined by

$$
H=-t \sum_{\langle i, j\rangle, \sigma}\left(c_{i \sigma}^{\dagger} c_{j \sigma}+c_{j \sigma}^{\dagger} c_{i \sigma}\right)+U \sum_{i} n_{i \uparrow} n_{i \downarrow}-\mu \sum_{i} n_{i},
$$

where $t$ is the hopping matrix element, $U$ is the onsite Coulomb repulsion, and $\mu$ is the chemical potential. The electron creation (annihilation) operator at site $i$ with spin $\sigma$ is represented by $c_{i \sigma}^{\dagger}\left(c_{i \sigma}\right), n_{i \sigma}=c_{i \sigma}^{\dagger} c_{i \sigma}$ is the electron occupation number with spin $\sigma$, and $n_{i}=n_{i \uparrow}+n_{i \downarrow}$. In the following, numerical data will be presented for $\langle n\rangle=0.875$ and $U=4 t$ and $8 t$. 
A nonmagnetic impurity located at the origin will be modelled by the onsite one-electron potential

$$
V_{\text {imp }}=V_{0} n_{0} .
$$

The value of $V_{0}$ will be taken to be $-20 t$, so that it is greater than both $U$ and the bandwidth. For this value of $V_{0}$, the electron occupation number of the impurity site is nearly 2 . In addition, the local magnetic moment defined by

$$
m(\mathbf{r})=\sqrt{\left\langle\left[m^{z}(\mathbf{r})\right]^{2}\right\rangle},
$$

where $m^{z}\left(\mathbf{r}_{i}\right)=n_{i \uparrow}-n_{i \downarrow}$, is close to zero at the impurity site $\mathbf{r}=(0,0)$. So, for this value of $V_{0}$, the impurity site is nearly doubly occupied and magnetically inert. Hence, one would expect that this model captures some of the physical properties of nonmagnetic impurities in strongly correlated systems, even though this form of bare impurity potential is a very simple one.

The Knight shift of the various nuclear sites is determined by the magnetic susceptibility $\chi$ through the relation

$$
k\left(\mathbf{r}_{i}\right)=\sum_{j} \chi\left(\mathbf{r}_{i}, \mathbf{r}_{j}, i \omega_{m}=0\right),
$$

where $j$ sums over the whole lattice. ${ }^{12}$ For instance, if the hyperfine coupling for the nuclear spin $\mathbf{I}_{i}$ at site $\mathbf{r}_{i}$ is $A \mathbf{I}_{i}$ - $\mathbf{S}_{i}$, then the corresponding Knight shift is given by $\left(\gamma_{e} / 2 \gamma_{n}\right) A k\left(\mathbf{r}_{i}\right)$, where $\gamma_{e}$ and $\gamma_{n}$ are the electronic and the nuclear gyromagnetic ratios. Here, the staggered magnetic susceptibility $\chi$ is defined by

$$
\chi\left(\mathbf{r}_{i}, \mathbf{r}_{j}, i \omega_{m}\right)=\int_{0}^{\beta} d \tau e^{i \omega_{m} \tau}\left\langle m_{i}^{-}(\tau) m_{j}^{+}(0)\right\rangle,
$$

where $\omega_{m}=2 m \pi T$ is the Bose Matsubara frequency $m_{i}^{+}$ $=c_{i \uparrow}^{\dagger} c_{i \downarrow}, m_{i}^{-}=c_{i \downarrow}^{\dagger} c_{i \uparrow}$, and $m_{i}^{-}(\tau)=e^{H \tau} m_{i}^{-} e^{-H \tau}$. The expectation value $\langle\cdots\rangle$ is evaluated with respect to the Hamiltonian of the impure system, which is given by $H+V_{\text {imp }}$. Note that for the translationally invariant pure system, $k(\mathbf{r})$ reduces to the uniform magnetic susceptibility $\chi_{\text {pure }}(\mathbf{q}$ $\left.\rightarrow 0, i \omega_{m}=0\right)$. In addition to these, results on the local magnetic moment $m(\mathbf{r})$ will be shown. Note that the sitedependent electron density $\langle n(\mathbf{r})\rangle$ and $m(\mathbf{r})$ were previously calculated. ${ }^{10}$ Here, these quantities are reproduced for comparisons with $k(\mathbf{r})$ and the effective impurity potential.

The transferred hyperfine coupling between the nuclear spin of a ${ }^{7} \mathrm{Li}$ impurity ${ }^{7} \mathbf{I}$ and the electronic spins in the $\mathrm{CuO}_{2}$ planes can be described by

$$
C^{7} \mathbf{I} \cdot \sum_{i=1}^{4} \mathbf{S}_{i}
$$

where $C$ is the hyperfine coupling constant and $i$ sums over the four nearest-neighbor $\mathrm{Cu}$ sites of the ${ }^{7} \mathrm{Li}$ impurity. In this case, the Knight shift for ${ }^{7} \mathrm{Li}$ is

$$
{ }^{7} K=\frac{1}{2}\left(\frac{\gamma_{e}}{{ }^{7} \gamma_{n}}\right) C 4 k[\mathbf{r}=(1,0)],
$$

so that the temperature dependence of ${ }^{7} \mathrm{~K}$ is determined by $k(\mathbf{r})$ evaluated at the nearest-neighbor site of the impurity $\mathbf{r}=(1,0)$. The measurements of the ${ }^{7} \mathrm{Li}$ Knight shift and of the ${ }^{89} \mathrm{Y}$ Knight shift at the first and the second nearestneighbor Y sites of the impurity determine $k(\mathbf{r})$ in the vicinity of the impurity. ${ }^{2,3,5}$ The analysis of the ${ }^{7} \mathrm{Li}$ and ${ }^{89} \mathrm{Y}$ Knight shift data on $\mathrm{Li}$ - and $\mathrm{Zn}$-substituted $\mathrm{YBa}_{2} \mathrm{Cu}_{3} \mathrm{O}_{6+x}$ concluded that $k(\mathbf{r})$ develops giant oscillations as $T$ decreases. ${ }^{5,12}$ The $T$-dependent line broadening of the ${ }^{63} \mathrm{Cu}$ NMR spectra in $\mathrm{Zn}$-substituted $\mathrm{YBa}_{2} \mathrm{Cu}_{3} \mathrm{O}_{6.7}$ has been also attributed to the development of a staggered polarization of the electronic spins, when a uniform magnetic field is applied. ${ }^{7}$ The QMC data presented here show that it is the $\mathrm{AF}$ correlations which are responsible for the pattern in $k(\mathbf{r})$.

In addition to the magnetic properties, the single-particle properties around the impurity will be studied. The singleparticle Green's function is defined by

$$
G\left(\mathbf{r}_{i}, \mathbf{r}_{j}, i \omega_{n}\right)=-\int_{0}^{\beta} d \tau e^{i \omega_{n} \tau}\left\langle c_{i \sigma}(\tau) c_{j \sigma}^{\dagger}(0)\right\rangle,
$$

where $\omega_{n}=(2 n+1) \pi T$ is a Fermi Matsubara frequency. Here, first the site-dependent electron density $\langle n(\mathbf{r})\rangle$ will be discussed. In order to understand the $\mathbf{r}$ dependence of $\langle n(\mathbf{r})\rangle$, the effective impurity potential will be extracted from the QMC data on $G$ of the impure system and $G_{U}$ of the pure Hubbard system. The Dyson's equation relating $G$ and $G_{U}$ is

$$
\begin{aligned}
G\left(\mathbf{r}, \mathbf{r}^{\prime}, i \omega_{n}\right)= & G_{U}\left(\mathbf{r}, \mathbf{r}^{\prime}, i \omega_{n}\right) \\
& +\sum_{\mathbf{r}_{1}, \mathbf{r}_{2}} G_{U}\left(\mathbf{r}, \mathbf{r}_{1}, i \omega_{n}\right) T\left(\mathbf{r}_{1}, \mathbf{r}_{2}, i \omega_{n}\right) \\
& \times G\left(\mathbf{r}_{2}, \mathbf{r}^{\prime}, i \omega_{n}\right),
\end{aligned}
$$

where $T\left(\mathbf{r}, \mathbf{r}^{\prime}, i \omega_{n}\right)$ is the effective impurity scattering matrix. Because of the Coulomb correlations, $T\left(\mathbf{r}, \mathbf{r}^{\prime}, i \omega_{n}\right)$ becomes extended in real space. ${ }^{9}$ Here, the diagonal component of $T$ is defined as the effective impurity potential

$$
V_{\mathrm{eff}}\left(\mathbf{r}, i \omega_{n}\right)=T\left(\mathbf{r}, \mathbf{r}, i \omega_{n}\right) .
$$

In Sec. IIIC, the spatial structure of $T\left(\mathbf{r}, \mathbf{r}^{\prime}, i \omega_{n}\right)$ will be discussed and results on $V_{\text {eff }}(\mathbf{r}, i \pi T)$ will be shown.

\section{NUMERICAL DATA}

\section{A. Knight shift}

The following data were obtained for electron filling $\langle n\rangle$ $=0.875$ and an $8 \times 8$ lattice with periodic boundary conditions. First, results will be shown for the $U=4 t$ case at temperatures between $0.25 t$ and 1.0t. In Fig. 1(a), $k(\mathbf{r})$ is plotted as a function of $r=|\mathbf{r}|$ in units of the lattice spacing $a$. The impurity is located at the origin at $\mathbf{r}=(0,0)$. For instance, in this notation, $r=1$ corresponds to $( \pm 1,0)$ and $(0, \pm 1)$ sites whereas $r=\sqrt{2}$ corresponds to $( \pm 1, \pm 1)$ and $( \pm 1, \mp 1)$. In addition, here $k(\mathbf{r})$ is shown in units of $t^{-1}$ and $t$ is taken to be unity. 

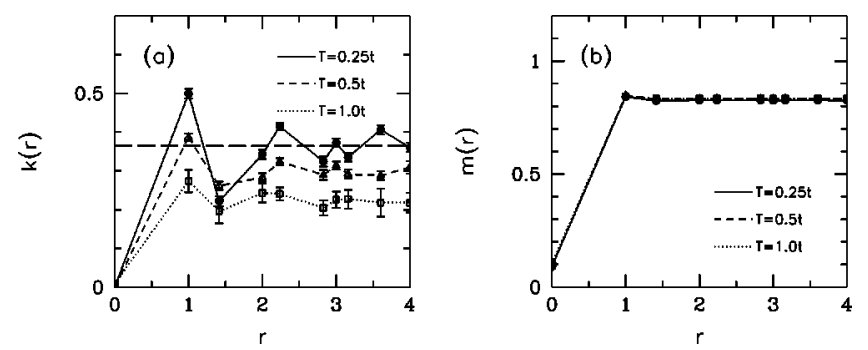

FIG. 1. (a) Knight shift response function $k(\mathbf{r})$ versus $r=|\mathbf{r}|$ away from the impurity at various temperatures. Here, $r$ is plotted in units of the lattice spacing. These results are for $U=4 t$ and $\langle n\rangle=0.875$ on an $8 \times 8$ lattice. The long-dashed horizontal line denotes the value of $k(\mathbf{r})$ for the pure system at $T=0.25 t$. (b) Local magnetic moment $m(\mathbf{r})=\sqrt{\left\langle\left[m^{z}(\mathbf{r})\right]^{2}\right\rangle}$ versus $r$ for the same parameters as in (a).

In Fig. 1(a), it is seen that $k(\mathbf{r})$ vanishes at the impurity site. This is because the strong impurity potential $V_{0}$ $=-20 t$ overcomes the electron-electron repulsion $U$ and binds an up-spin and a down-spin electron at $\mathbf{r}=(0,0)$, so that the impurity site becomes magnetically inert. In the vicinity of the impurity, $k(\mathbf{r})$ exhibits oscillations, which grow as the temperature decreases. The horizontal long-dashed line in Fig. 1(a) represents the value of $k(\mathbf{r})$ for the pure system at $T=0.25 t$. Hence, away from the impurity, $k(\mathbf{r})$ goes to its value for the pure system, which is the uniform magnetic susceptibility. Since these calculations are carried out at high temperatures, the uniform susceptibility does not yet exhibit the temperature-independent Pauli behavior. These data on $k(\mathbf{r})$ show that the nonmagnetic impurity strongly influences the zero-frequency magnetic correlations around it.

In Fig. 1(b), results on $m(\mathbf{r})$ are plotted in the same way as in Fig. 1(a). Here, it is seen that, between $T=0.25 t$ and $1.0 t, m(\mathbf{r})$ is very weakly dependent on the temperature. At $\mathbf{r}=(0,0), m(\mathbf{r})$ has a small value. Comparing with Fig. 1(a), it is observed that the oscillations in $k(\mathbf{r})$ are stronger than those in $m(\mathbf{r})$. Note that in both $k(\mathbf{r})$ and $m(\mathbf{r})$, the maximum occurs at $\mathbf{r}=(1,0)$. The NMR experiments also find that $k(\mathbf{r})$ has its maximum value at $\mathbf{r}=(1,0)$.

In order to understand the origin of the oscillatory structure in $k(\mathbf{r})$, it is useful to consider the Fourier transform of $\chi\left(\mathbf{r}, \mathbf{r}^{\prime}, i \omega_{m}\right)$ defined by

$$
\chi\left(\mathbf{q}, \mathbf{q}^{\prime}, i \omega_{m}\right)=\sum_{\mathbf{r}, \mathbf{r}^{\prime}} e^{-i\left(\mathbf{q} \cdot \mathbf{r}-\mathbf{q}^{\prime} \cdot \mathbf{r}^{\prime}\right)} \chi\left(\mathbf{r}, \mathbf{r}^{\prime}, i \omega_{m}\right) .
$$

This quantity is related to the Fourier transform $k(\mathbf{q})$ $=\Sigma_{\mathbf{r}} e^{-i \mathbf{q} \cdot \mathbf{r}} k(\mathbf{r})$ through

$$
k(\mathbf{q})=\chi\left(\mathbf{q}, \mathbf{q}^{\prime}=0, i \omega_{m}=0\right) .
$$

In Fig. 2(a), $-k(\mathbf{q})$ is plotted at different temperatures as a function of $\mathbf{q}$ along various cuts in the Brillouin zone. For comparison, in Fig. 2(b) the diagonal susceptibility

$$
\chi(\mathbf{q})=\chi\left(\mathbf{q}, \mathbf{q}, i \omega_{m}=0\right)
$$
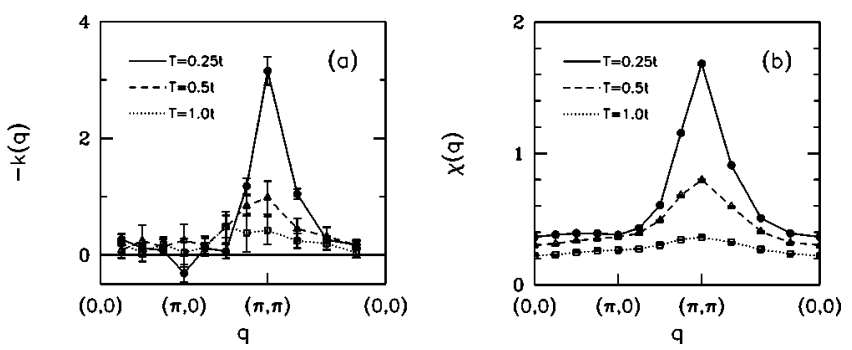

FIG. 2. (a) Wave vector dependence of the Knight shift response function $k(\mathbf{q})$ along various cuts in the Brillouin zone. (b) Diagonal magnetic susceptibility $\chi(\mathbf{q})=\chi\left(\mathbf{q}, \mathbf{q}, i \omega_{m}=0\right)$ versus $\mathbf{q}$. These results are for $U=4 t,\langle n\rangle=0.875$, and an $8 \times 8$ lattice at various temperatures.

versus $\mathbf{q}$ is plotted. In these figures, both $k(\mathbf{q})$ and $\chi(\mathbf{q})$ are plotted in units of $t^{-1}$. Here, it is seen that $-k(\mathbf{q})$ develops a sharp peak at $\mathbf{q}=(\pi, \pi)$, which closely follows the development of the peak in $\chi(\mathbf{q})$ and, hence, of the AF correlations in the system. This relation between $-k(\mathbf{q})$ and the $\mathrm{AF}$ correlations becomes more clear if the following RPA expression for $\chi\left(\mathbf{q}, \mathbf{q}^{\prime}, i \omega_{m}\right)$ is considered, ${ }^{11,12}$

$$
\begin{aligned}
\chi\left(\mathbf{q}, \mathbf{q}^{\prime}, i \omega_{m}\right)= & \chi_{0}\left(\mathbf{q}, \mathbf{q}^{\prime}, i \omega_{m}\right) \\
& +\bar{U} \sum_{\mathbf{q}^{\prime \prime}} \chi\left(\mathbf{q}, \mathbf{q}^{\prime \prime}, i \omega_{m}\right) \chi_{0}\left(\mathbf{q}^{\prime \prime}, \mathbf{q}^{\prime}, i \omega_{m}\right),
\end{aligned}
$$

where $\bar{U}$ is the effective irreducible vertex in the particlehole channel and $\chi_{0}\left(\mathbf{q}^{\prime \prime}, \mathbf{q}^{\prime} ; i \omega_{m}\right)$ is the susceptibility of the impure $U=0$ system. For $\mathbf{q}^{\prime}=0$ and $i \omega_{m}=0$, one obtains

$$
k(\mathbf{q})=k_{0}(\mathbf{q})+\bar{U} \sum_{\mathbf{q}^{\prime \prime}} \chi\left(\mathbf{q}, \mathbf{q}^{\prime \prime}, 0\right) k_{0}\left(\mathbf{q}^{\prime \prime}\right),
$$

where $k_{0}(\mathbf{q})$ is for the impure $U=0$ system. This expression shows that the peak in $k(\mathbf{q})$ at $\mathbf{q}=(\pi, \pi)$ is coupled to the AF fluctuations. The QMC data seen in Figs. 2(a) and 2(b) show that this is true for the real system also and that it is the AF correlations which cause the development of the giant oscillations in $k(\mathbf{r})$.

At this point, it is necessary to discuss the physical meaning of the $\mathbf{q}$ dependence of $k(\mathbf{q})$. The QMC data and the Knight shift experiments show that $k(\mathbf{q})$ peaks at $\mathbf{q}$ $\sim(\pi, \pi)$. Since $k(\mathbf{q})$ is equivalent to the off-diagonal susceptibility $\chi\left(\mathbf{q}, \mathbf{q}^{\prime}=0, i \omega_{m}=0\right)$, this result means that the scattering of the AF spin fluctuations with $\sim(\pi, \pi)$ momentum transfer is one of the important effects of the nonmagnetic impurities. This then gives information about the response of the system in the magnetic channel to a perturbation in the density channel.

It is useful to compare the structure in $k(\mathbf{r})$ with the sitedependent electron density $\langle n(\mathbf{r})\rangle$. Figure 3 shows $\langle n(\mathbf{r})\rangle$ versus $r$ for the same parameters as in Figs. 1 and 2. At the impurity site $\mathbf{r}=(0,0)$, the electron density is nearly equal to two and it is not included in this graph. There are oscillations in $\langle n(\mathbf{r})\rangle$ which decay as one moves away from the impurity. 


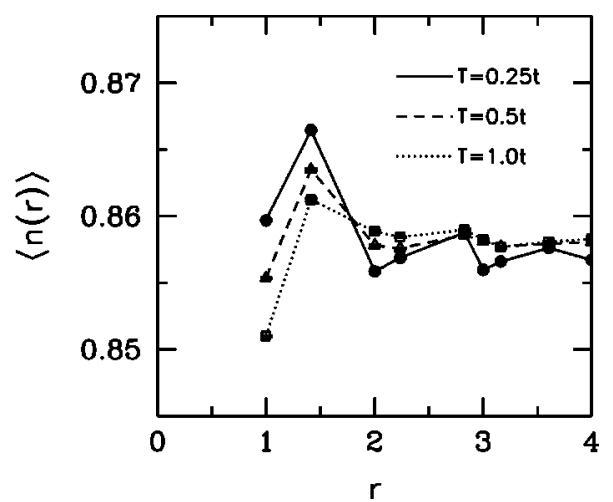

FIG. 3. Site-dependent electron density $\langle n(\mathbf{r})\rangle$ versus $r$ plotted in the same way as in Fig. 1. These data are for $U=4 t,\langle n\rangle$ $=0.875$, and an $8 \times 8$ lattice at various temperatures.

For sites close to the impurity and especially for $\mathbf{r}=(1,0)$ and $(1,1),\langle n(\mathbf{r})\rangle$ increases as $T$ decreases.

Figures 4(a) through 4(d) show QMC data on $k(\mathbf{r})$, $-k(\mathbf{q}), \chi(\mathbf{q})$ and $\langle n(\mathbf{r})\rangle$ for the stronger coupling case of $U=8 t$. These data are plotted in the same way as for $U$ $=4 t$ above. The structures in $k(\mathbf{r}), k(\mathbf{q}), \chi(\mathbf{q})$ are similar to what have been seen for $U=4 t$, only the amplitudes differ. When $U$ increases to $8 t$, the impurity site remains doubly occupied, however, the structure in $\langle n(\mathbf{r})\rangle$ changes. Compared to the $U=4 t$ case, here $\langle n[\mathbf{r}=(1,0)]\rangle$ has a bigger enhancement over the background. As it will be seen in Sec. IIIC, this has to do with the fact that $V_{\text {eff }}(\mathbf{r}, i \pi T)$ at $\mathbf{r}$ $=(1,0)$ is more attractive for $U=8 t$.

These calculations for $U=4 t$ and $8 t$ were repeated for an electron filling of 0.94 . In this case, the system has longerrange AF fluctuations. Consequently, $k(\mathbf{r})$ is more enhanced and has stronger oscillations. However, the structures in $k(\mathbf{r}), k(\mathbf{q}), \chi(\mathbf{q})$, and $\langle n(\mathbf{r})\rangle$ are similar to what have been shown for the $\langle n\rangle=0.875$ case. Because of space limitations these results will not be presented here.

\section{B. Comparison with the RPA analysis of the Knight shift data}

The QMC data on the Knight shifts are at high temperatures to be directly compared with the experimental data. However, the real-space structure of $k(\mathbf{r})$ and $\langle n(\mathbf{r})\rangle$ calculated with QMC are in good agreement with the results of the RPA analysis of the experimental data. ${ }^{12}$ In the RPA approach, an effective impurity potential which is extended in real space and independent of frequency

$$
V_{\mathrm{eff}}=V_{0} n_{0}+V_{1} \sum_{i=1}^{4} n_{i}
$$

was used to model the nonmagnetic impurity. The onsite component $V_{0}$ was set to a large attractive value, and the extended component $V_{1}$, which is acting at the four sites neighboring the impurity, was used as a fitting parameter. Both $V_{0}$ and $V_{1}$ were taken to be real valued. Then, the magnetic susceptibility and $k(\mathbf{r})$ were calculated by using an RPA-like approach for treating the Coulomb correlations. The magnetic susceptibility $\chi_{0}$ of the impure $U=0$ system entering the RPA expression was calculated by including the self-energy and the vertex corrections due to the impurity scattering. It was found that for an effective quasiparticle bandwidth of $1 \mathrm{eV}$, the ${ }^{7} \mathrm{Li}$ Knight shift data on the optimally doped $\mathrm{YBa}_{2} \mathrm{Cu}_{3} \mathrm{O}_{6+x}$ can be fitted over a wide temperature range by using $V_{1}=-0.15 t$.

In this approach, the magnitudes of the quantities such as $k(\mathbf{r})$ or the value of $V_{1}$ used for fitting the data depends on the effective bandwidth, and it is difficult to know what the exact value of the effective bandwidth should be in such a model. In addition, there are uncertainties in the values of the hyperfine couplings. For these reasons, what should really be compared with is the pattern of the oscillations in $k(\mathbf{r})$ and $\langle n(\mathbf{r})\rangle$, and not so much the magnitudes.

In Fig. 5(a), $k(\mathbf{r})$ obtained by fitting the ${ }^{7} \mathrm{Li}$ Knight shift

${ }^{7} \mathrm{~K}$ in optimally doped $\mathrm{YBa}_{2} \mathrm{Cu}_{3} \mathrm{O}_{6+x}$ with ${ }^{7} \mathrm{Li}$ impurities is
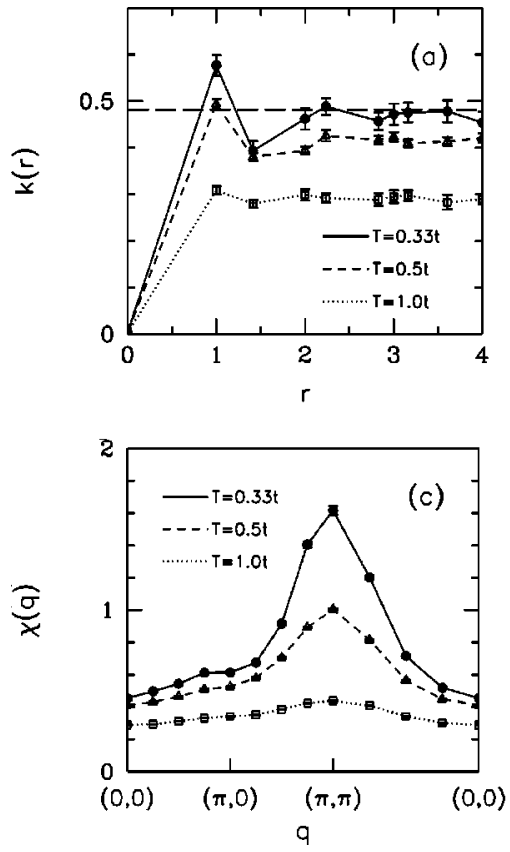
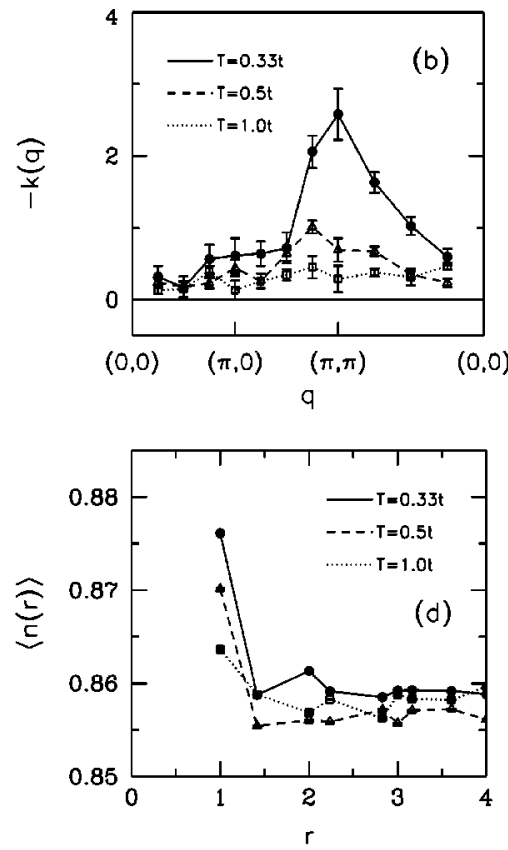

FIG. 4. (a) $k$ (r) versus $r$, (b) $-k(\mathbf{q})$ versus $\mathbf{q}$, (c) $\chi(\mathbf{q})$ versus $\mathbf{q}$ and (d) $\langle n(\mathbf{r})\rangle$ versus $r$ for $U$ $=8 t,\langle n(\mathbf{r})\rangle=0.875$, and an $8 \times 8$ lattice at various temperatures. In (a), the long-dashed horizontal line denotes the value of $k(\mathbf{r})$ for the pure system at $T=0.33 t$. 

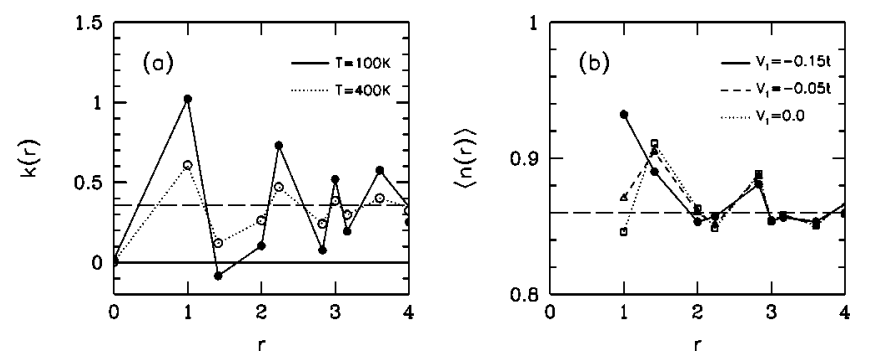

FIG. 5. (a) Knight shift response function $k(\mathbf{r})$ versus $r$ at 100 and $400 \mathrm{~K}$ obtained for the optimally doped YBCO with $\mathrm{Li}$ impurities. This result was obtained within an RPA analysis of the experimental data by using $V_{1}=-0.15 t$. Here, $V_{1}$ is the value of the effective impurity potential at the sites neighboring the impurity. (b) $\langle n(\mathbf{r})\rangle$ versus $r$ obtained from the same RPA calculations at $100 \mathrm{~K}$ for different values of $V_{1}$. In these figures, the long-dashed horizontal lines represent the values of $k(\mathbf{r})$ and $\langle n(\mathbf{r})\rangle$ for the pure system at $T=100 \mathrm{~K}$.

shown at 100 and $400 \mathrm{~K}$. In this calculation, the ${ }^{7} \mathrm{Li}$ hyperfine coupling was taken to be $1.8 \times 10^{-20} \mathrm{erg}$, corresponding to $0.85 \mathrm{kOe} / \mu_{B}$. It is useful to compare $k(\mathbf{r})$ seen in Fig. 5(a) with the QMC data presented in Figs. 1(a) and 4(a). Here, it is seen that as $T$ decreases, the QMC data develop real-space structure which is similar to the results of the RPA analysis.

It is difficult to compare the experimental data with the QMC calculations directly, but it would still be useful to discuss the temperature range where the QMC calculations have been performed. The single-band parameters which are considered to be appropriate for the cuprates are $t$ $\sim 0.45 \mathrm{eV}$ and $U$ of order $12 t .{ }^{16}$ It is difficult to reach low temperatures with large $U / t$. Hence, here calculations have been carried out for $U=8 t$ and $4 t$. For $U=8 t$, QMC data have been shown down to $T=0.33 t$. For this value of $U$, the magnetic exchange $J \sim 4 t^{2} / U$ is $0.5 t$ and, hence, $T=0.33 t$ corresponds to two thirds of $J$, which is too high to make comparisons with the experiments. For the intermediate coupling $U=4 t$ case, the lowest $T$ where QMC data are shown is $0.25 t$. In this case, the magnetic exchange $J \sim t$, and $T$ $=0.25 t$ corresponds to $J / 4$. In fact, the QMC data on $k(\mathbf{r})$ for $U=4 t$ and $T=0.25 t$, which are shown by the filled circles in Fig. 1(a), compare well with the RPA fits to the experimental data at $400 \mathrm{~K}$, shown by the empty circles in Fig. 5(a). This is encouraging, however, the expression $J$ $\sim 4 t^{2} / U$ for the magnetic exchange is valid in the strong coupling limit. So, it is difficult to compare the QMC data directly with the experiments. On the other hand, studying how $k(\mathbf{r})$ evolves with $U / t$ and $T / t$ shows what to expect for the Knight shifts at low temperatures in the strong coupling limit.

Within the same RPA framework and using an effective bandwidth of $1 \mathrm{eV}$, the Knight shift data on ${ }^{7} \mathrm{Li}$ and ${ }^{89} \mathrm{Y}$ were also fitted for the underdoped $\mathrm{YBa}_{2} \mathrm{Cu}_{3} \mathrm{O}_{6+x}$ with nonmagnetic impurities. In this case, it was found that, as $T$ decreases from 400 to $100 \mathrm{~K}, k[\mathbf{r}=(1,0)]$ increases from $0.8 t^{-1}$ to $2.1 t^{-1}$, whereas $k[\mathbf{r}=(1,1)]$ goes from $0.04 t^{-1}$ to $-0.8 t^{-1}$. This clearly demonstrates the severe effect of the nonmagnetic impurity on the zero-frequency magnetic corre- lations in the underdoped cuprates. At the temperatures where they are performed, the QMC calculations also find that the oscillations in $k(\mathbf{r})$ are filling dependent. For example, for $U=4 t$ and $T=0.25 t, k[\mathbf{r}=(1,0)]$ increases by about $10 \%$, when $\langle n\rangle$ varies from 0.875 to 0.94 . On the other hand, if $\langle n\rangle$ goes from 0.875 to 0.80 , then $k(\mathbf{r}=(1,0))$ decreases by about $30 \%$. In this paper, QMC results are shown for $\langle n\rangle=0.875$, because, for this value of the filling and at the temperatures where these calculations are carried out, the system has short-range AF correlations which are not weak, and their effects are visible.

Next, comparisons are made for the local electron density. Figure $5(\mathrm{~b})$ shows $\langle n(\mathbf{r})\rangle$ versus $\mathbf{r}$ obtained from the same RPA analysis at $100 \mathrm{~K}$ for different values of $V_{1}$. The oscillations seen in this figure are stronger than in the QMC data, which were obtained at higher temperatures. Here, it is seen that as $V_{1}$ becomes more attractive, the electron density at $\mathbf{r}=(1,0)$ increases. Comparison with the QMC data in Figs. 3 and 4(d) implies that the effective impurity potential at $\mathbf{r}$ $=(1,0)$ is more attractive for $U=8 t$ than for $4 t$. In the next subsection, it will be seen that this is indeed the case.

\section{Effective impurity interaction}

In order to understand the spatial structure of $\langle n(\mathbf{r})\rangle$, it is necessary to discuss the effective scattering matrix $T\left(\mathbf{r}, \mathbf{r}^{\prime}, i \omega_{n}\right)$, which can be obtained from

$$
\mathbf{T}\left(i \omega_{n}\right)=\mathbf{G}_{U}^{-1}\left(i \omega_{n}\right)-\mathbf{G}^{-1}\left(i \omega_{n}\right) .
$$

Here, $\mathbf{T}\left(i \omega_{n}\right)$ is a matrix of which $(i, j)$ th element is $T\left(\mathbf{r}_{i}, \mathbf{r}_{j}, i \omega_{n}\right), \mathbf{T}^{-1}$ is the inverse of $\mathbf{T}$, and similarly for $\mathbf{G}$ and $\mathbf{G}_{U}$. In the following, QMC results will be shown for the lowest Matsubara frequency $\omega_{n}=\pi T$. For $\mathbf{r}$ and $\mathbf{r}^{\prime}$ more than one or two lattice spacings away from the impurity, $T\left(\mathbf{r}, \mathbf{r}^{\prime}, i \pi T\right)$ is small in the parameter regime the QMC calculations were performed. The diagonal terms of the scattering matrix $T$ are represented by $V_{\text {eff }}\left(\mathbf{r}, i \omega_{n}\right)$. At the impurity site $\mathbf{r}=(0,0), V_{\text {eff }}(\mathbf{r}, i \pi T)$ is reduced from its bare value of $-20 t$ due to the Coulomb correlations, and it also has an imaginary part. For $U=4 t$ and $T=0.25 t, V_{\text {eff }}(\mathbf{r}, i \pi T)$ at $\mathbf{r}$ $=(0,0)$ is $-18 t+i 0.6 t$, while for $U=8 t$ and $T=0.5 t$, it is $-15 t+i 2 t$. Away from the impurity site, $V_{\mathrm{eff}}(\mathbf{r}, i \pi T)$ is largest at $( \pm 1,0)$ and $(0, \pm 1)$. In addition, the effective scattering matrix has large matrix elements between the impurity site and the sites neighboring it. Hence, the presence of the impurity renormalizes the bare hopping matrix elements between the impurity and its neighbors. The off-diagonal terms such as $T\left[\mathbf{r}=(1,0), \mathbf{r}^{\prime}=(0,1), i \pi T\right]$ are small compared to $V_{\text {eff }}[\mathbf{r}=(1,0), i \pi T]$.

Figure 6(a) shows the real-space structure of the real and the imaginary parts of $V_{\text {eff }}(\mathbf{r}, i \pi T)$ for $U=4 t$ and $T$ $=0.25 t$, and Fig. 6(b) shows the temperature dependence of $V_{\text {eff }}[\mathbf{r}=(1,0), i \pi T]$. Here, it is seen that $V_{\text {eff }}$ at $\mathbf{r}=(1,0)$ is weakly attractive. The results for $U=8 t$ are shown in Figs. $7(\mathrm{a})$ and $7(\mathrm{~b})$. In this case, $V_{\text {eff }}[\mathbf{r}=(1,0), i \pi T]$ has a bigger value. At $T=0.5 t$, its real and imaginary parts are of order $-t / 4$. This is why $\langle n[\mathbf{r}=(1,0)]\rangle$ for $U=8 t$ is more enhanced over the background than for $4 t$. 

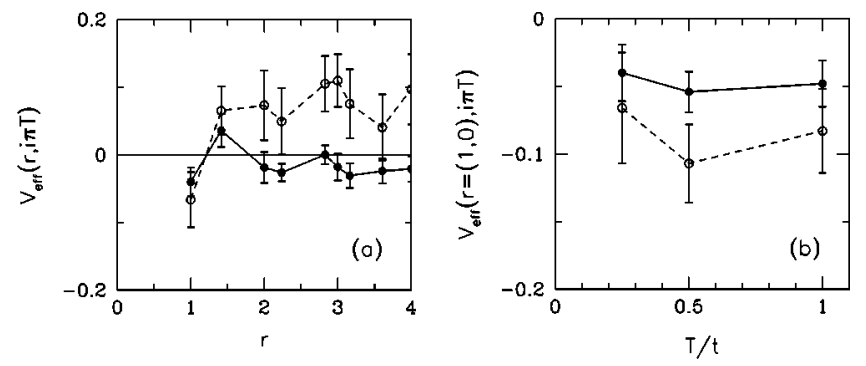

FIG. 6. (a) Effective impurity potential $V_{\text {eff }}(\mathbf{r}, i \pi T)$ versus $r$ at $T=0.25 t$. (b) $V_{\text {eff }}(\mathbf{r}, i \pi T)$ at $\mathbf{r}=(1,0)$ versus $T$. Here, the filled circles denote the real part of $V_{\text {eff }}$, and the open circles denote the imaginary part. These results are for $U=4 t$ and $\langle n\rangle=0.875$.

In previous analysis of the NMR data, ${ }^{12}$ an RPA-type approximation was used for treating the Coulomb correlations and the effective impurity potential was taken to be extended in space with the form given by Eq. (16), but it was assumed that $V_{\text {eff }}$ is real-valued and independent of frequency. Within this model, the fitting of the Knight shift data required that $V_{\text {eff }}$ is weakly attractive at the sites neighboring the impurity. The QMC data presented in Figs. 6 and 7 show that the real part of $V_{\text {eff }}(\mathbf{r}, i \pi T)$ at $\mathbf{r}=(1,0)$ is attractive, which is in agreement with the RPA fitting of the data. The QMC calculations also find that $T\left(\mathbf{r}, \mathbf{r}^{\prime}, i \omega_{n}\right)$ depends on frequency, and, in addition, it has an imaginary component. Since $\operatorname{Im} T\left(\mathbf{r}, \mathbf{r}^{\prime}, i \omega_{n}\right)$ is odd in $\omega_{n}, \operatorname{Im} T\left(\mathbf{r}, \mathbf{r}^{\prime}, i \pi T\right)$ should vanish as the temperature goes to zero. In order to investigate the effects of $V_{\text {eff }}$ having an imaginary component, the RPA calculations were repeated using $V_{1}\left(i \omega_{n}\right)=-0.15 t[1$ $\left.+i \operatorname{sgn}\left(\omega_{n}\right)\right]$ instead of $V_{1}=-0.15 t$ in Eq. (16), and keeping all other parameters the same in the calculations. It was found that the imaginary component of $V_{1}$ affects the magnitudes of $k(\mathbf{r})$ and $\langle n(\mathbf{r})\rangle$, but the patterns in these quantities remain similar. These comparisons with the QMC data on $T\left(\mathbf{r}, \mathbf{r}^{\prime}, i \omega_{n}\right)$ support the form of $V_{\text {eff }}$, Eq. (16), used in the RPA analysis of the Knight shift experiments.

\section{CONCLUSIONS}

The substitution of nonmagnetic impurities into the layered cuprates provides useful information about the manybody physics of these materials. In particular, the nonmagnetic impurities constitute a perturbation in the density channel, and the Knight shift measurements determine the real-space resolved response of the system to this perturbation. In the NMR experiments on ${ }^{7} \mathrm{Li},{ }^{89} \mathrm{Y}$, and ${ }^{63} \mathrm{Cu}$ nuclei in $\mathrm{YBa}_{2} \mathrm{Cu}_{3} \mathrm{O}_{6+x}$ with nonmagnetic impurities, it has been found that, when a uniform magnetic field is applied, the electronic spins in $\mathrm{CuO}_{2}$ planes polarize to form a magnetization with an oscillatory pattern. ${ }^{2-7}$ In order to understand the origin of this behavior, here QMC data have been presented for one nonmagnetic impurity in the 2D Hubbard
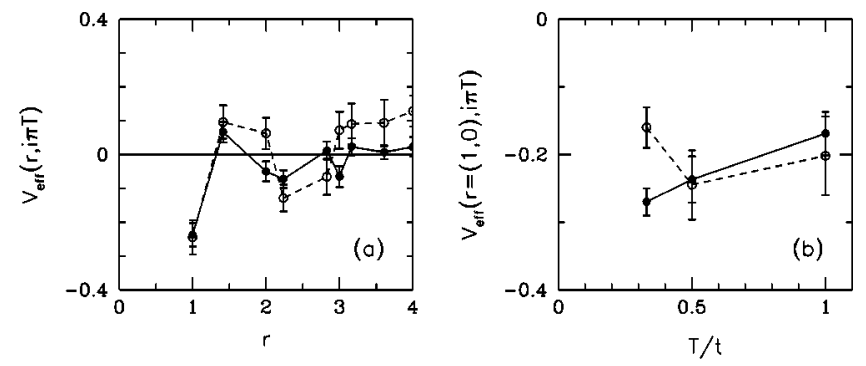

FIG. 7. (a) Effective impurity potential $V_{\text {eff }}(\mathbf{r}, i \pi T)$ versus $r$ at $T=0.5 t$. (b) $V_{\text {eff }}(\mathbf{r}, i \pi T)$ at $\mathbf{r}=(1,0)$ versus $T$. Here, the filled circles denote the real part of $V_{\text {eff }}$, and the open circles denote the imaginary part. These results are for $U=8 t$ and $\langle n\rangle=0.875$.

model. These QMC data show that, as the temperature is lowered and the AF fluctuations grow, giant oscillations develop in the Knight shift response around the impurity. The pattern of these oscillations and of the electron density were discussed here. In addition, the effective impurity potential extracted from the QMC data was shown. Since these results are restricted to high temperatures, it is not possible to compare directly with the NMR experiments. For this reason, the QMC data were compared with the results of the previous RPA analysis of the NMR data. It was found that, as $T$ decreases, the patterns which develop in the Knight shift response and the local electron density are in agreement with those found in the RPA analysis by fitting the experimental data.

In spite of these, a number of open questions remain. For instance, it is not known whether, within the 2D Hubbard model with only nearest-neighbor hopping, a fit to the perfect Curie-Weiss $T$ dependence of the ${ }^{7} \mathrm{Li}$ Knight shift will be obtained at low $T$ between 100 and $400 \mathrm{~K}$. Perhaps a second-near-neighbor hopping or other terms are required for describing the pure material. It is also possible that the bare impurity potential is more involved than the simple form of Eq. (2) used here. Nevertheless, the QMC data presented here clearly show the role of the AF fluctuations in producing the giant oscillations in the Knight shift response.

\section{ACKNOWLEDGMENTS}

The author gratefully acknowledges helpful discussions with H. Alloul, J. Bobroff, W. Hanke, and M. Imada. The author also thanks J. Bobroff for helpful comments about the manuscript. This work was supported by the Turkish Academy of Sciences through the GEBIP program (Grant No. EA-TUBA-GEBIP/2001-1-1). The author thanks the Institute for Solid State Physics at the University of Tokyo and the Institute for Theoretical Physics at the University of Würzburg for their hospitality. The numerical computations reported in this paper were performed at the Center for Information Technology at Koç University. 
${ }^{1}$ G. Xiao, M.Z. Cieplak, J.Q. Xiao, and C.L. Chien, Phys. Rev. B 42, 8752 (1990).

${ }^{2}$ A.V. Mahajan, H. Alloul, G. Collin, and J.-F. Marucco, Phys. Rev. Lett. 72, 3100 (1994).

${ }^{3}$ J. Bobroff, W.A. MacFarlane, H. Alloul, P. Mendels, N. Blanchard, G. Collin, and J.-F. Marucco, Phys. Rev. Lett. 83, 4381 (1999).

${ }^{4}$ P. Mendels, J. Bobroff, G. Collin, H. Alloul, M. Gabay, J.-F. Marucco, N. Blanchard, and B. Grenier, Europhys. Lett. 46, 678 (1999).

${ }^{5}$ A.V. Mahajan, H. Alloul, G. Collin, and J.-F. Marucco, Eur. Phys. J. B 13, 457 (2000).

${ }^{6}$ W.A. MacFarlane, J. Bobroff, H. Alloul, P. Mendels, N. Blanchard, G. Collin, and J.-F. Marucco, Phys. Rev. Lett. 85, 1108 (2000).

${ }^{7}$ M.-H. Julien, T. Feher, M. Horvatic, C. Berthier, O.N. Bakharev, P. Segransan, G. Collin, and J.-F. Marucco, Phys. Rev. Lett. 84,
3422 (2000).

${ }^{8}$ D. Poilblanc, D.J. Scalapino, and W. Hanke, Phys. Rev. Lett. 72, 884 (1994); Phys. Rev. B 50, 13020 (1994).

${ }^{9}$ W. Ziegler, D. Poilblanc, R. Preuss, W. Hanke, and D.J. Scalapino, Phys. Rev. B 53, 8704 (1996).

${ }^{10}$ W. Ziegler, H. Endres, and W. Hanke, Phys. Rev. B 58, 4362 (1998).

${ }^{11}$ N. Bulut, Phys. Rev. B 61, 9051 (2000).

${ }^{12}$ N. Bulut, Physica C 363, 260 (2001).

${ }^{13}$ Y. Ohashi, Phys. Rev. B 66, 054522 (2002).

${ }^{14}$ R. Blankenbecler, D.J. Scalapino, and R.L. Sugar, Phys. Rev. D 24, 2278 (1981).

${ }^{15}$ S.R. White, D.J. Scalapino, R.L. Sugar, E.Y. Loh, J.E. Gubernatis, and R.T. Scalettar, Phys. Rev. B 40, 506 (1989).

${ }^{16}$ M.S. Hybertsen, E.B. Stechel, M. Schluter, and D.R. Jennison, Phys. Rev. B 41, 11068 (1990). 